\title{
Tecnura
}

INVESTIGACIÓN

\section{Metodología para el monitoreo estructural y patológico de viviendas afectadas por deslizamientos}

\author{
Structural and pathological monitoring methodology \\ for homes affected by landslides
}

Sergio Giovanny Valbuena Porras , César Augusto García-Ubaque², Martha Alejandra Granados Soler ${ }^{3}$

Fecha de recepción: 17 de septiembre de 2016

Fecha de aceptación: 15 de febrero de 2017

Cómo citar: Valbuena P., S.G.; García-Ubaque, C.A. y Granados S., M.A. (2017). Metodología para el monitoreo estructural y patológico de viviendas afectadas por deslizamientos. Revista Tecnura, 21(52), 79-87. doi: 10.14483/udistrital.jour.tecnura.2017.2.a06

\section{Resumen}

Contexto: En los últimos sesenta años los procesos de urbanización se han incrementado considerablemente. En gran parte de ellos, el número de habitantes de los denominados tugurios asciende a más del $50 \%$ de la población y, adicionalmente, se encuentran localizados en zonas de riesgo.

Objetivo: Determinar y evaluar la progresión mensual del índice de daño (ID) durante un periodo de cuatro meses a un grupo de viviendas ubicado en zona de ladera en el sector Altos de la Estancia en Bogotá, Colombia.

Método: Las viviendas fueron clasificadas de acuerdo con su sistema estructural en seis grupos: mampostería confinada (grupo A), mampostería estructural (grupo B), mampostería no reforzada (grupo C), materiales provisionales (grupo D), pórticos en concreto reforzado (grupo E) y viviendas prefabricadas (grupo F) y se les aplicó la metodología propuesta por el Fondo para la Prevención y Atención de Emergencias (FOPAE).
Resultados: En todos los grupos se observó un incremento constante en el ID; sin embargo, el grupo $\mathrm{C}$ presentó los valores más altos en el último muestreo: $2,6 \%$ de las viviendas (ID alto) y $0,87 \%$ (ID muy alto). En los demás grupos el ID observado fue menor y no se encontraron viviendas con registros clasificados como muy altos.

Conclusiones: De acuerdo con los resultados, las viviendas construidas en mampostería no reforzada presentan un mayor grado de deterioro en periodos cortos de tiempo en comparación a aquellas construidas con otros sistemas estructurales.

Palabras clave: deslizamiento, monitoreo estructural, índice de daño, vulnerabilidad, viviendas.

\begin{abstract}
Context: In the last sixty years urbanization processes have increased considerably. In many of them, the number of inhabitants of the so-called "slums" amounts to more than $50 \%$ of the population. In addition, they are located in areas at risk.
\end{abstract}

1 Ingeniero civil, magíster en Economía. Profesor Asistente Facultad Tecnológica de la Universidad Distrital Francisco José de Caldas. Bogotá, Colombia. Contacto: svalbuena@udistrital.edu.co

2 Ingeniero civil, magíster en Ingeniería Civil, doctor en Ingeniería. Profesor Asociado de la Facultad Tecnológica de la Universidad Distrital Francisco José de Caldas. Bogotá, Colombia. Contacto: cagarciau@udistrital.edu.co

3 Tecnóloga en Construcciones Civiles, candidata a Ingeniera Civil. Universidad Distrital Francisco José de Caldas. Bogotá, Colombia. Contacto: magranadoss@correo.udistrital.edu.co 
Objective: To determine and evaluate the monthly progression of the Damage Index (ID, in Spanish) of a group of houses located in a hillside area in the "Altos de la Estancia" sector in Bogotá, Colombia, during a period of four months.

Method: The houses were classified according to their structural system in six groups: confined masonry (group A); structural masonry (group B); unreinforced masonry (group C); provisional materials (group D); reinforced concrete porches (group E); and prefabricated houses (group F). And we applied the methodology proposed by the Fund for the Prevention and Attention of Emergencies (FOPAE).
Results: There was a constant increase in the ID of all groups. However, group C presented the highest values in the last sampling $(2.6 \%$ of the households had high ID and $0.87 \%$ very high). In the other groups the observed ID was lower and no houses were found with records classified as very high.

Conclusions: According to the results, dwellings built in unreinforced masonry show a greater degree of deterioration in short periods of time compared to those constructed with other structural systems.

Keywords: sliding, structural monitoring, damage index, vulnerability, houses.

\section{INTRODUCCIÓN}

En los últimos sesenta años el proceso de urbanización, tanto en América Latina como en el resto del mundo, se ha incrementado considerablemente, de tal manera que más de la mitad de la población total del planeta, es decir aproximadamente 3300 millones de individuos, viven en áreas urbanas y se espera que esta cifra ascienda a $6000 \mathrm{mi}-$ llones para el 2050 (United Nations 2014). En gran parte de estos centros urbanos, particularmente en aquellos que pertenecen a los países en vías de desarrollo, el número de habitantes de los denominados tugurios asciende a más del $50 \%$ de la población. En la mayoría de casos, estos carecen del acceso a vivienda, agua y saneamiento, educación o servicios de salud y se encuentran en zonas de riesgo. Comprender esta situación implica, en primera instancia, entender la urbanización como un proceso social e histórico, en el cual las condiciones espacio-temporales propias de cada zona, han traído consigo no solo cambios demográficos y territoriales sino transformaciones en las prácticas sociales y en la manera como los individuos significan los lugares.

En Colombia, si bien hacia la primera mitad del siglo XX la mayoría de la población del país vivía en zonas rurales, la llegada de la idea de la modernidad que se encontró estrechamente asociada a los ambientes urbanos junto con la construcción de imaginarios sociales -desde los cuales se generó un anhelo por la inserción del país a las sociedades más adelantadas- y la ola de violencia que azotó las zonas rurales durante la segunda mitad del siglo fueron factores determinantes que suscitaron el desarrollo de fuertes corrientes migratorias masivas del campo a la ciudad, las cuales acarrearon importantes modificaciones en los centros urbanos (De la Cueva 1993). Este panorama no ha sido ajeno a la ciudad de Bogotá, la cual al configurarse como capital, se ha convertido en la mayor receptora de población migrante (Bolivar y Erazo, 2013), situación que se evidencia en la diseminación de numerosos asentamiento humanos que en muchos casos se localizan en zonas expuestas a riesgos por inestabilidad de los terrenos, inundaciones, mala disposición de residuos, entre otros (Lattes 2000; Charvériat 2000).

Como ejemplo de lo anterior se encuentra el sector Altos de la Estancia, ubicado en los cerros suroccidentales del Distrito Capital, en la localidad de Ciudad Bolívar. Esta zona era una extensa área rural en la que predominaba el bosque nativo hasta mediados del siglo XX. Sin embargo, fue deforestada para el desarrollo de actividades agrícolas y ganaderas. Desde comienzos de la década 
de 1990 experimentó el inicio e intensificación de un proceso de urbanización, caracterizado por la presencia de viviendas que carecían de cualquier tipo de parámetro técnico constructivo, sumado a la ausencia de obras de urbanismo mínimas como redes de acueducto y alcantarillado. Esta situación dio paso a la aparición de vertimientos de aguas servidas y fugas de agua potable que, junto al auge de la explotación minera, desestabilizaron la ladera (Rojas 2004).

En agosto de 1997 se registró el primer movimiento de tierra en esta zona, el cual demandó la reubicación de dos familias, la intervención de la quebrada la Carbonera y la regulación de la actividad minera. No obstante, y a pesar de las medidas tomadas, la inestabilidad del cerro continuaba, ocasionando los dos eventos de mayor magnitud que han sido registrados en esta zona: el primero, en abril del año 2000, el cual afectó a 567 viviendas y causó la declaración de esta área como zona de alto riesgo; y el segundo, ocurrido en mayo de 2002, y que formó una grieta de 30 metros, averiando 804 casas (Fopae, 2014). Dichos deslizamientos que se enmarcan entre los cuerpos hídricos La Carbonera, Santo Domingo y Santa Rita, se encuentran activos, son de tipo compuesto, complejo y múltiple, con un área de afectación directa aproximada de 73,17 hectáreas.

Debido al impacto en la población y al riesgo que implica la localización de viviendas en zonas de riesgo a nivel mundial, se han elaborado una serie de instrumentos y metodologías para la investigación, seguimiento y monitoreo de las amenazas. Entre estos destacan las normas australianas (AGS, 2007) y europea (JTC-1 2008). Por su parte, Schuster y Kockelman (1966) proponen una serie de principios generales y metodologías enfocados en la reducción de amenazas de deslizamiento utilizando sistemas de prevención, los cuales demandan tanto la participación de los entes gubernamentales como de las comunidades. No obstante, en muchos casos la eliminación total de los problemas no es posible mediante métodos preventivos, sino que requiere establecer medidas de control y monitoreo (Suárez, 1998). En Colombia se han desarrollado varios trabajos de tipo metodológico para la evaluación y el control de riesgo por deslizamiento, entre los cuales se pueden mencionar los de la Dirección de Prevención y Atención de Emergencias (2000); Ojeda, Castro, Valencia y Fonseca (2002); Ingeocim (1998), y Yamin, Ghesquiere, Cardona y Ordaz, (2013), incluidos en la Guía metodológica para estudios de amenaza, vulnerabilidad y riesgo por movimientos en masa (Ávila et al., 2015).

Para el caso de Altos de la Estancia, debido a que actualmente existen viviendas localizadas en inmediaciones de una zona catalogada como de alto riesgo no mitigable, se pretendió estimar la vulnerabilidad física de las construcciones, determinando si esta se debe a la exposición al fenómeno amenazante, o si por el contrario se encuentra más relacionada con deficiencias en las estructuras físicas, es decir los materiales, procesos constructivos y cumplimiento de normas. Así, pues, se realizó el monitoreo estructural de acuerdo con la metodología propuesta por la empresa Consultores en Ingeniería y Medio Ambiente $\mathrm{Cl}$ Ambiental SAS, la cual a su vez se encuentra soportada en la metodología propuesta por el Consorcio Altos de la Estancia. Este instrumento incluye un proceso de caracterización inicial de las viviendas, seguido por una valoración periódica del daño estructural que busca determinar los cambios en ID. Este índice permite evaluar la progresión de las lesiones estructurales encontradas, con el objetivo de poder determinar las acciones que deben adoptarse en cada una de ellas.

De acuerdo con la información previa es necesario determinar si las construcciones localizadas en esta área están más expuestas al daño físico, por lo que estas construcciones presentarían un ID más alto que el usual. El objetivo de este estudio es identificar y analizar los cambios físicos que pueden haber sido experimentados por las edificaciones, con relación a la variación del ID y sus posibles causas. 


\section{Zona de estudio}

Las campañas de monitoreo y seguimiento estructural se realizaron en 484 edificaciones localizadas en los barrios Santa Viviana, Santo Domingo, El Espino I, El Espino, Ismael Perdomo, Mirador de la Estancia, Perdomo Alto y Sierra Morena, ubicados en la zona periférica del polígono denominada "Altos de la Estancia", en la ciudad de Bogotá. Cada vivienda se inspeccionó de manera individual. Para el análisis e identificación de estas se buscó el Código Homologado de Identificación Predial CHIP correspondiente a cada predio, suministrado por el Catastro Nacional.

\section{METODOLOGÍA}

\section{Caracterización de viviendas}

Con el fin de facilitar la clasificación de las viviendas presentes en la zona de estudio, se recopiló la siguiente información:

- Información general de la construcción: dirección y localización, el código CHIP y algunos datos de los residentes.

- Características de la edificación, de acuerdo con las siguientes variables:

\section{Sistema estructural}

1. Sistema de pórticos.

2. Mampostería estructural.

3. Mampostería confinada.

4. Mampostería no reforzada.

5. Prefabricado.

6. Materiales no convencionales: cualquier tipo de sistema que emplee materiales y configuraciones que no se encuentre dentro de los anteriores.

Calidad de la construcción: se refiere al nivel de cumplimento de las especificaciones técnicas mínimas de construcción: buena, regular, mala.

Altura de la edificación: número de niveles presentes.
Las viviendas fueron clasificadas en seis grupos, según los siguientes parámetros: grupo A: viviendas con mampostería confinada, grupo B: viviendas con mampostería estructural, grupo C: viviendas con mampostería no reforzada (simple), grupo D: materiales provisionales, grupo E: pórticos en concreto reforzado y grupo F: viviendas prefabricadas.

\section{Evaluación del índice de daño (ID)}

Las viviendas seleccionadas fueron inspeccionadas visualmente en campo. Para facilitar la recolección y el registro de los datos obtenidos, se estructuró el "Formato de evaluación del índice de daño de viviendas" (figura 1), el cual se utilizó para evaluar de forma rutinaria y progresiva el estado en que se encontraban las viviendas mediante la observación detallada, con prolongaciones de tiempo mensuales durante un periodo de cuatro meses.

Este formato incluye tres bloques de información:

1. Identificación de la vivienda y generalidades: información necesaria para identificar a qué vivienda pertenece el formato y facilitar su búsqueda.

2. Índice de daño de la vivienda: para el cálculo del índice de daño se recurre al método heurístico, que permite combinar lo cualitativo con lo cuantitativo. De este modo, se definieron las siguientes variables para calcular la vulnerabilidad estructural de las viviendas:

a. Condición o estado de la edificación: en la inspección visual se califica de manera cualitativa la condición en la que se encuentran cuatro elementos de las edificaciones: la cimentación, los muros, el entrepiso y la cubierta. Esta calificación se debe hacer según los criterios presentados en la tabla 1, en una escala de bueno, regular y malo. A esta apreciación se le asigna un valor numérico definido según su incidencia en la edificación. 


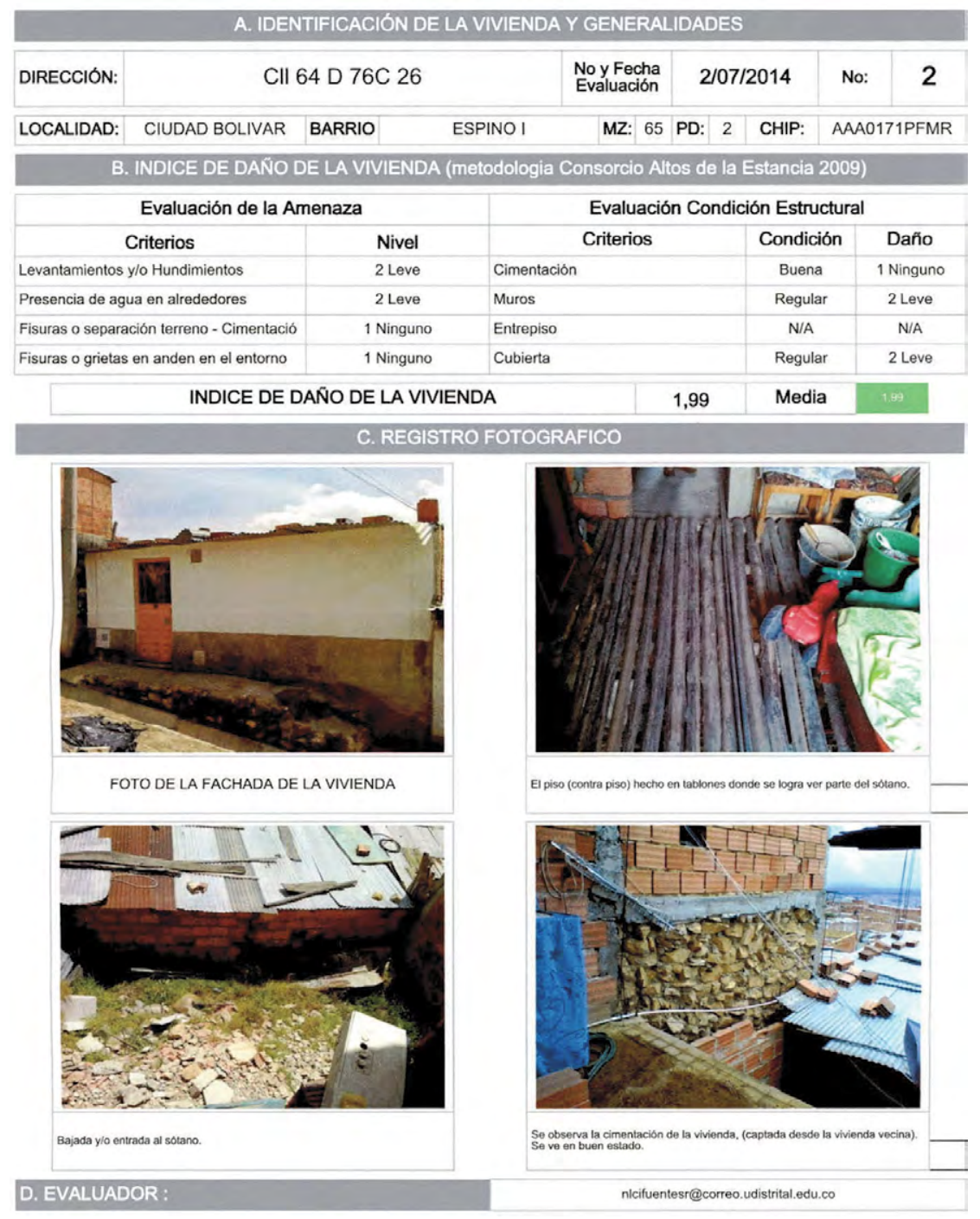

Figura 1. Formato de evaluación del índice de daño de viviendas

Fuente: elaboración propia.

Tabla 1. Criterios de evaluación del primer factor

\begin{tabular}{cc}
\hline Condición Inicial & Primer factor (F1) \\
\hline Buena & 1 \\
\hline Regular & 1,05 \\
\hline Mala & 1,1 \\
\hline
\end{tabular}

Fuente: elaboración propia.
En caso de que el elemento no pueda ser evaluado, se califica como N/A (no aplica) y como valor de ponderación de 1,1 .

b. Daño estructural

Se califica el daño presente en los elementos estructurales evaluados anteriormente, utilizando igualmente una calificación cualitativa, asociada a un criterio y a una ponderación, como se presenta en la tabla 2. 
Tabla 2. Criterios para estimar la magnitud del daño

\begin{tabular}{cc}
\hline Magnitud del daño & $\begin{array}{c}\text { Magnitud del } \\
\text { daño (D) }\end{array}$ \\
\hline Si no existe daño & 1 \\
\hline Si el daño es leve & 2 \\
\hline Si el daño es moderado & 3 \\
\hline Si el daño es fuerte & 4 \\
\hline Si el daño es severo & 6 \\
\hline
\end{tabular}

Fuente: elaboración propia.

Como elementos de control y medición se realizó la colocación de testigos (portaobjetos) en las grietas de alguna de las viviendas, con el fin de medir el avance de estas, y la dirección de movimiento en términos de agrietamiento e incrementos, y dirección de estos.

3. Registro fotográfico: con el fin de llevar un registro visual del estado de las viviendas, se llevó un recuento fotográfico detallado y concordante de cada una durante las cuatro visitas.

\section{Calculó del índice de daño (ID)}

El ID se obtiene a partir de los de los ponderados de las calificaciones de los daños, afectados por el factor de mayoración de la condición de cada elemento, mediante el siguiente procedimiento:

1. Se define un primer factor (F1), que es igual al valor numérico asociado a la evaluación de la condición de cada elemento estructural (tabla 1).

2. Se calcula un segundo factor para la cimentación (Fc2), los muros (Fm2), el entrepiso (Fe2), la cubierta (Fcu2) (ecuación (1)).

$$
F 2=F 1 * D
$$

3. Se calcula un tercer factor (F3), de acuerdo con la cantidad de pisos que tiene la vivienda

a. Si el número de pisos es mayor que 1 (ecuación (2)).

$$
F 3=(0,6 * F e 2)+(0,4 * F c u) .
$$

b. Si el número de pisos es 1 (ecuación (3)).

$$
F 3=(\text { Fcu } 2) \text {. }
$$

c. Se calcula el ID que presenta la vivienda usando la siguiente fórmula (ecuación (4)).

$I D=(0,1 * F c 2)+(0,5 * F m 2)+(0,4 * F 3)$

Por último, se determina dentro de cuál de los siguientes rangos de la tabla 3 se encuentra el ID y obtener un valor cualitativo de la vulnerabilidad de cada vivienda.

Tabla 3. Rango del índice de daño (ID)

\begin{tabular}{cc}
\hline $\begin{array}{c}\text { Rango del índice de daño } \\
\text { (ID) }\end{array}$ & Vulnerabilidad \\
\hline $\mathrm{ID}<1$ & Muy baja \\
\hline $1 \geq \mathrm{ID}<2$ & Baja \\
\hline $2 \geq \mathrm{ID}<3$ & Media \\
\hline $3 \geq \mathrm{ID}<4$ & Alta \\
\hline $4 \geq \mathrm{ID}<5$ & Muy alta \\
\hline
\end{tabular}

Fuente: elaboración propia.

\section{RESULTADOS}

De las 484 viviendas inspeccionadas, $158(32,64$ $\%)$ se incluyeron en el grupo $A ; 1(0,21 \%)$ en el grupo B; 231 (47,73\%) en el grupo C; 8 (1,65\%) en el grupo D; 53 (10,95\%) en el grupo E, y 33 $(6,82 \%)$ en el grupo F. Del total de viviendas, durante la primera evaluación se encontraron 452 con vulnerabilidad baja (93,39\%), 26 con vulnerabilidad media $(5,37 \%)$ y 6 con vulnerabilidad alta $(1,24 \%)$. Al final del estudio se observó que la vulnerabilidad fue baja en 386 viviendas $(79,75 \%)$, media para $80(16,53 \%)$, alta para 15, alta para $16(3,51 \%)$ y muy alta para $2(0,41 \%)$. En la tabla 4 , se muestra progresión de la vulnerabilidad durante el periodo evaluado.

Con relación a los sistemas estructurales, inicialmente en el grupo A se observó que 150 viviendas $(94,94 \%)$ presentaron vulnerabilidad 
Tabla 4. Progresión del índice de daño en las viviendas evaluadas

\begin{tabular}{ccccc}
\hline \multicolumn{5}{c}{ Índice de daño (ID) } \\
\hline Campaña & Bajo & Medio & Alto & Muy alto \\
\hline 1 & 452 & 26 & 6 & \\
\hline 2 & 432 & 43 & 9 & 1 \\
\hline 3 & 415 & 57 & 11 & 2 \\
\hline 4 & 386 & 80 & 16 & \\
\hline
\end{tabular}

Fuente: elaboración propia.

baja; 7 (4,43\%), media, y $1(0,63 \%)$, alta. En el grupo B, 1 vivienda (100\%) presentó vulnerabilidad baja. En el grupo C, 211 (91,34\%) presentaron vulnerabilidad baja; 17 (7,36\%), media, y 3 $(1,30 \%)$, alta. En el grupo D,

$7(87,50 \%)$, presentaron vulnerabilidad baja y $1(12,50 \%)$, alta. En el grupo E, $52(98,11 \%)$ presentaron vulnerabilidad baja y $1(1,89 \%)$, media. Por último, en el grupo E, 31 (93,94\%) presentaron vulnerabilidad baja; 1 (3,03\%), media, y 1 $(3,03 \%)$, alta. Al final del estudio, en el grupo A, $131(82,91 \%)$ viviendas presentaron vulnerabilidad baja; 24 (15,19\%), media, y 3 (1,90\%), alta. En el grupo B, 1 (100\%) vivienda presentó vulnerabilidad media. En el grupo C, 178 (77,06 \%) presentaron vulnerabilidad baja; 45 (19,48\%), media; $6(2,60 \%)$, alta, y $2(0,87 \%)$, muy alta. En el grupo D, $6(75 \%)$ viviendas presentaron vulnerabilidad baja y 2 (25\%), alta. En el grupo E, 47 (88,68 $\%$ ) presentaron vulnerabilidad baja y 5 (9,43\%), media y $1(1,89 \%)$, alta. Por último, en el grupo $\mathrm{F}$, $24(72,73 \%)$ viviendas presentaron vulnerabilidad baja; 5 (15,15\%), media, y $4(12,12 \%)$, alta. En la tabla 5 se puede observar la evolución de la vulnerabilidad por grupo durante el periodo evaluado.

Para el caso del grupo A, las viviendas con vulnerabilidad baja decrecieron en un 3,93 \% en los cuatro meses del estudio; en los grupos B y D este decrecimiento fue del $0,21 \%$; en el C (en donde se registró la mayor variación) fue de un 6,82 \%; en el grupo E fue de 1,03 \%, y en el F, de 4,45\%. Por su parte, la vulnerabilidad media y alta aumentó en cuatro de los grupos: para el $\mathrm{A}$, en un 3,51\% y un $0,41 \%$; para el grupo $C$, en un $5,79 \%$ y un $0,62 \%$; para el grupo $E$, en un $0,83 \%$ y un 0,21 $\%$, y para el grupo $F$, en un $0,83 \%$ y un $0,62 \%$. En el caso del grupo B, el aumento solo fue en la vulnerabilidad media con un $0,21 \%$ y el grupo D en la vulnerabilidad alta con un 0,21\%.

Tabla 5. Evolución del índice de daño (ID) durante el periodo evaluado

\begin{tabular}{lcccccccccccccc}
\hline \multicolumn{1}{c}{ Campaña } & & $\mathbf{1}$ & & & $\mathbf{2}$ & & & & & & & & \\
\hline Vulnerabilidad & $\mathbf{B}$ & $\mathbf{M}$ & $\mathbf{A}$ & $\mathbf{B}$ & $\mathbf{M}$ & $\mathbf{A}$ & $\mathbf{B}$ & $\mathbf{M}$ & $\mathbf{A}$ & $\mathbf{M A}$ & $\mathbf{B}$ & $\mathbf{M}$ & $\mathbf{A}$ & $\mathbf{M A}$ \\
\hline Grupo A & 151 & 7 & 0 & 141 & 17 & 0 & 137 & 20 & 1 & 0 & 131 & 24 & 3 & 0 \\
\hline Grupo B & 1 & 0 & 0 & 1 & 0 & 0 & 0 & 1 & 0 & 0 & 0 & 1 & 0 & 0 \\
\hline Grupo C & 211 & 17 & 3 & 204 & 24 & 3 & 195 & 31 & 4 & 1 & 178 & 45 & 6 & 2 \\
\hline Grupo D & 7 & 0 & 1 & 6 & & 2 & 6 & & 2 & 0 & 6 & 0 & 2 & 0 \\
\hline Grupo E & 52 & 1 & & 52 & 1 & & 50 & 3 & 0 & 0 & 24 & 5 & 4 & 0 \\
\hline Grupo F & 31 & 1 & 1 & 28 & 1 & 4 & 27 & 2 & 4 & 0 & 24 & 5 & 4 & 0 \\
\hline Total general & 435 & 26 & 5 & 432 & 43 & 9 & 415 & 57 & 11 & 1 & 386 & 80 & 16 & 2 \\
\hline
\end{tabular}

$\mathbf{B}=$ baja; $M=$ media; $A=$ alta; $M A=$ muy alta.

Fuente: elaboración propia. 


\section{DISCUSIÓN}

Mediante este estudio se quiso realizar una inspección preliminar de las viviendas en cuanto a los elementos que configuran su estructura, con miras a determinar las afectaciones encontradas en estas y establecer un proceso de seguimiento para dichas lesiones. De este modo, fue posible obtener un diagnóstico mediante el cual se evaluaron los inmuebles en función de su índice de daño y se clasificaron según su sistema estructural. Este parámetro fue escogido como categoría de agrupación, debido a que el número de pisos estaba implícito en el cálculo del ID, y no se tuvo en cuenta la calidad de la construcción debido que la evaluación de la misma depende considerablemente de la percepción del evaluador y la metodología no define unos criterios unificados para la calificación cualitativa de la misma.

No obstante, la aplicación de esta metodología se vio limitada debido a que, por tratarse de asentamientos informales que no han seguido ningún patrón de planificación, estos modelos de autoconstrucción en su mayoría carecen tanto de un diseño arquitectónico como estructural, a lo que se suma la incorporación de materiales de distinta índole como madera, tejas, latas, lonas, piezas de demolición, entre otros; factores que no son considerados dentro de los criterios de evaluación establecidos en la metodología. Por otro lado, en gran parte de los predios que debían ser inspeccionados no fue posible acceder debido a las negativas de sus propietarios, situación que suscita que en estos casos la evaluación se llevara a cabo solo de manera exterior.

\section{CONCLUSIONES}

Es importante señalar que, en el diseño de metodologías para la caracterización y evaluación de viviendas, debe reconocerse la existencia de una serie de prácticas constructivas que difieren en muchos aspectos de las construcciones formales, dando lugar a que sea necesario incluir otra serie de variables en la formulación de aquellas, en cuanto a la incorporación de los elementos que caracterizan los contextos espacio-temporales de estos inmuebles y desde los cuales se puede explicar su morfología.

Esta evaluación de daño, en principio, se le adjudica a la falla, pero no se puede desconocer la influencia de las prácticas constructivas; por consiguiente, en instrumentos futuros se deben considerar indicadores que permitan incorporar y evaluar de manera adecuada estos factores.

\section{REFERENCIAS}

Australian Geomechanics Society (AGS) (2007). Guidelines for Landslide Susceptibility, Hazard and Risk Zoning for Land Use Planning. Aust Geomech, 42(1), 13-36. doi:10.1016/j.enggeo.2008.03.022.

Ávila, G.E.; Cubillos, C.E.; Granados, A.E.; Medina, E.; Rodríguez, E.; Rodríguez, C.E. y Ruiz, G.L. (2015). Guía metodológica para estudios de amenaza, vulnerabilidad y riesgo por movimientos en masa del Servicio Geológico Colombiano. Bogotá: Servicio Geológico Colombiano.

Bolivar, T. y Erazo, J. (2013). Los lugares del hábitat y la inclusión. Recuperado de: http://www2.sgc.gov.co/ Noticias/archivos/Guia-Metodologica-Servicio-Geologico.aspx

Charvériat, C. (2000). Natural disasters in Latin America and the Caribbean: An overview of risk. Recuperado de: http://www.econstor.eu/bitstream/10419/88044/1/idb-wp_434.pdf.

Clark, D. (1998). Interdependent Urbanization in an Urban World: An Historical Overview. The Geographical Journal, 164(1), 85-95. doi:10.2307/3060547. The Royal Geographical Society (with the Institute of British Geographers).

Ayala, J. (2014) Monitoreo de viviendas ubicadas en la zona de Altos de la Estancia en la localidad de Ciudad Bolívar. Tesis de Grado Tecnología en Construcciones Civiles, Universidad Distrital Francisco José de Caldas, Bogotá, Colombia.

De la Cueva, A.A. (1993). Los asentamientos populares y el orden jurídico en la urbanización periférica 
de américa latina. Revista Mexicana de Sociología, 55(3), 133. doi:10.2307/3540926.

Dirección de Prevención y Atención de Emergencias del Distrito (DPAE) (2000). Términos de referencia para estudios de riesgos por inestabilidad del terreno. Bogotá.

Fondo de Atención y Prevención de Emergencias (Fopae) (2014). Lo que la tierra se llevó. Altos de La Estancia: sueños de vida digna. Bogotá.

Frisbie, P. (1975). The Human Consequences of Urbanisation: Divergent Paths in the Urban Experience of the Twentieth Century. American Journal of Sociology, 80(4), 1022-1024. doi:10.2307/2777217. The University of Chicago Press.

Ingeocim Ltda. (1998). Zonificación por inestabilidad del terreno para diferentes localidades en la ciudad de Santafé de Bogotá. Bogotá.

Joint Technical Committee (JTC-1) (2008). Guidelines for Landslide Susceptibility, Hazard and Risk Zoning for Land Use Planning. Engineering Geology, 102, 85-98. -doi:10.1016/j.enggeo.2008.03.009.

Lattes, A. (2001). Población urbana y urbanización en América Latina, en Carrión F. (Ed.), La ciudad construida, urbanismo en América Latina (pp. 4976). Quito, Ecuador: FLACSO Ecuador-Junta de Andalucía.

Ojeda, J.; Castro, E.; Valencia, A. y Fonseca, S. (2002). Evaluación del riesgo por fenómenos de remoción en masa: guía metodológica. Bogotá: Ingeominas, Corporación Autónoma Regional del Valle del Cauca, Escuela Colombiana de Ingeniería.

Rojas, Z.C. (2004). Sin memoria muero: memorias de un proceso de resistencia: Altos de la Estancia, Ciudad Bolívar 2004. Bogotá.

Schuster, R.L. y Kockelman, W.J. (1966). Principles of Landslide Hazard Reduction. En: A.K. Turner y L.R. Schuster (eds.). Landslides Investigation and Mitigation, Special Report 247 (pp. 91-105). Washington, EE. UU.: National Research Council, Transportation Research Board.

Suárez D., J. (1998). Deslizamientos y estabilidad de taludes en zonas tropicales. Bucaramanga: Ingeniería de Suelos Ltda.

United Nations (2014). World Urbanization Prospects, the 2014 Revision. Nueva York: Department of Economic and Social Affairs. doi:10.4054/ DemRes.2005.12.9.

Yamin, L.E.; Ghesquiere, F.; Cardona, O.D. y Ordaz, M.G. (2013). Modelación probabilista para la gestión del riesgo de desastres: el caso de Bogotá, Colombia. Washington DC: World Bank, Universidad de los Andes. Recuperado de: http://documents. worldbank.org/curated/en/2013/07/18100020/ colombia-probabilistic-modeling-disaster-risk-management-modelacion-probabilista-para-la-gestion-del-riesgo-de-desastre.

\section{(c) $(1) \Theta$}

